\title{
On Models of Meteoroids Fragmentation in the Atmosphere
}

\author{
I.G. Brykina ${ }^{1}$, M.D. Bragin ${ }^{2,3}$, L.A. Egorova ${ }^{1}$ \\ ${ }^{1}$ Institute of Mechanics of Lomonosov Moscow State University, Moscow, 119192, Russia \\ ${ }^{2}$ Keldysh Institute of Applied Mathematics, Moscow, 125047, Russia \\ ${ }^{3}$ Moscow Institute of Physics and Technology, Dolgoprudny, Moscow Region, 141701, Russia \\ shantii@mail.ru
}

\begin{abstract}
Different approaches are developed to modelling the destruction of cosmic bodies in the atmosphere into a large number of fragments. It is assumed that they first move with a common shock wave as a single body deformed by pressure forces. Four models of meteoroid disruption into a cloud of fragments are considered: two developed by the authors, and two generally accepted. The main differences between the models are showed. Using these models, numerical solutions of the meteor physics equations taking into account ablation are obtained to simulate the interaction of the Chelyabinsk meteoroid with the atmosphere. Solutions for different models are compared with each other and with observational data.

After meteoroid's fragments diverge over a long distance, their independent movement is considered. Formulas for the probability and cumulative distribution of fragments by mass are obtained using the results of experiments on the destruction of bodies under high-speed impact. The cumulative distribution is compared with the experimental data and also with the mass distributions of the recovered meteorites in the cases of a significant number of fragments found. The mass and luminosity of the disrupted meteoroid are determined by integration over all fragments using the probabilistic mass distribution as the initial one. The mass and velocity of each fragment are determined from the meteor physics equations.
\end{abstract}

Keywords: meteoroid, ablation, fragmentation. 
УДК 532.68

\title{
О моделях фрагментации метеороидов в атмосфере
}

\author{
И.Г. Брыкина ${ }^{1}$, М.Д. Брагин ${ }^{2,3}$, Л.А. Егорова ${ }^{1}$ \\ ${ }^{1}$ НИИ механики МГУ им. М.В. Ломоносова, Москва, 119192, Мичуринский пр., 1 \\ ${ }^{2}$ ИПМ им. М.В. Келдыша РАН, Москва, 125047, Миусская пл., 4 \\ ${ }^{3}$ Московский физико-технический институт, Долгопрудный, 141701, Институтский п., 7 \\ shantii@mail.ru
}

\begin{abstract}
Аннотация
Развиты разные подходы к моделированию разрушения космических тел в атмосфере на множество фрагментов. Предполагается, что сначала они движутся с общей ударной волной как единое тело, деформирующееся под действием сил давления. Рассмотрены четыре модели дробления метеороида на облако фрагментов: две, развитые авторами, и две общепринятые. Выявлены основные различия между моделями. С использованием этих моделей получены численные решения уравнений метеорной физики с учетом абляции для расчета взаимодействия Челябинского метеороида с атмосферой. Решения для разных моделей сравниваются друг с другом и с данными наблюдений.

После расхождения фрагментов на большое расстояние рассматривается независимое их движение. Для вероятностного и кумулятивного распределения фрагментов по массам получены формулы с использованием результатов экспериментов по разрушению тел при высокоскоростном ударе. Кумулятивное распределение сравнивается с экспериментальными данными и с распределениями по массам найденных метеоритов в случаях значительного числа обнаруженных осколков. Масса раздробленного метеороида и интенсивность свечения находятся интегрированием по всем фрагментам с использованием вероятностного распределения их по массам как начального. Масса и скорость каждого фрагмента определяются из уравнений метеорной физики.
\end{abstract}

Ключевые слова: метеороид, абляция, разрушение

\section{1. Введение}

Большинство входящих в атмосферу Земли космических тел разрушаются под действием аэродинамических нагрузок, возрастающих по мере проникновения в более плотные слои атмосферы. Разрушение - один из основных процессов, наряду с абляцией, оказывающих существенное влияние на взаимодействие космического тела с атмосферой. Космические тела имеют разный состав, скорость, структуру (включая наличие дефектов), размер, и разрушаются они по-разному. Об этом свидетельствуют разнообразные формы световых кривых метеороидов, построенных по наземным и спутниковым наблюдениям интенсивности их свечения вдоль траектории.

Поэтому существуют разные подходы к моделированию разрушения метеороидов. В одном предполагается, что при разрушении тела на множество фрагментов они движутся с общей ударной волной как единый объект до тех пор, пока фрагменты не разойдутся. Под действием сил давления облако фрагментов деформируется: сжимается в направлении движения и расширяется в поперечном. Этот подход, предложенный С.С. Григоряном [1], применялся позднее в разных вариациях во многих работах. В другом подходе считается, что фрагменты двигаются независимо со своей собственной ударной волной. Тело может дробиться на два-три или на множество фрагментов, дробление может быть одностадийным 
или прогрессивным, идея которого была предложена Б.Ю. Левиным [2]. При таком подходе надо знать распределение фрагментов по массам. В данной работе для моделирования на основе физической теории метеоров $[2,3]$ разных аспектов взаимодействия метеороида с атмосферой при дроблении его на множество фрагментов применялись оба подхода.

\section{2. Модели облака фрагментов, движущихся с общей ударной волной}

Предполагается, что до начала разрушения на высоте $h_{f}$ метеороид движется как единое тело (шар), затем он продолжает двигаться как облако фрагментов, паров и мелких быстро испаряющихся частиц, объединенных общей ударной волной. Полагается также, что такое облако под действием сил давления деформируется: сжимается в продольном движению направлении и расширяется в поперечном. Рассмотрим четыре модели дробления метеороида на облако фрагментов: две общепринятые - модель I Григоряна $[1,4]$ и модель II Хиллза и Годы [5], часто используемая в литературе, и две модели, предложенные авторами статьи - модель III [6] и IV - модификацию модели III.

\section{1. Разработанные модели фрагментации}

В модели III предполагаются два процесса: сплющивание раздробленного метеороида - трансформация шара в сфероид с отношением полуосей $b / a=k \geq 1$, и уменьшение его плотности из-за увеличения промежутков между фрагментами $-\delta=\delta_{e} / \gamma^{3}$, где $\delta$ и $\delta_{e}-$ текущая и начальная плотности. Имеем два параметра: сплющивания $k$ и уменьшения плотности $\gamma$.

Для параметра $\gamma$ получено соотношение в предположении, что скорость увеличения расстояния между фрагментами пропорциональна $\rho^{1 / 2}[7]$, где $\rho$ - плотность атмосферы

$$
\gamma=1+\frac{\rho^{1 / 2}-\rho_{f}^{1 / 2}}{\rho_{m}^{1 / 2}-\rho_{f}^{1 / 2}}\left(\gamma_{m}-1\right)
$$

Индексы $f, m$ относятся к величинам на высотах $h_{f}$ и пика светимости болида $h_{m}$. Полагаем, что $\gamma \leq 3$ [8], параметр $\gamma_{m}$ определяется приравниваем расчетной и наблюдаемой высот $h_{m}$.

Для радиуса миделева сечения $R_{S}$ получено уравнение с учетом распределения давления вдоль поверхности сфероида

$$
\frac{\mathrm{d} R_{S}}{\mathrm{~d} t}=\frac{1}{k^{1 / 2}}\left(\frac{\gamma^{3} \rho}{\delta_{e}}\right)^{1 / 2} V, \quad k=\frac{4 \pi \delta_{e}}{3} \frac{R_{S}^{3}}{M \gamma^{3}}
$$

Здесь $V$ и $M$ - скорость и масса метеороида в текущий момент времени; $t$ - время.

Модель IV отличается от модели III тем, что в ней ограничивается рост параметра $k$.

\section{2. Отличия разработанных моделей от общепринятых}

Уравнения для радиуса миделева сечения $R_{S}$ в моделях I и II получены без учета изменения формы метеороида (фактически для шара) и имеют одинаковую структуру

$$
\frac{\mathrm{d} R_{S}}{\mathrm{~d} t}=c\left(\frac{\rho}{\delta_{e}}\right)^{1 / 2} V,
$$

где $c=$ const : $c=1$ в модели I; $c=(7 / 2)^{1 / 2}$ в модели II. Уравнение (3) в случае прямолинейной траектории имеет аналитическое решение 


$$
R_{S}=R_{f}\left(1+\frac{2 c h^{*}}{\sin \theta \delta_{e}^{1 / 2} R_{f}}\left(\rho^{1 / 2}-\rho_{f}^{1 / 2}\right)\right)
$$

Здесь $\theta$ - угол входа метеороида в атмосферу по отношению к горизонту; $R_{f}$ - его радиус на высоте $h_{f} ; h^{*}=7$ км (шкала высот). Из соотношения (4) следует, что значение радиуса $R_{S}$ в моделях I и II полностью определяется только начальными параметрами: $\theta, \rho_{f}, R_{f}$ и $\delta_{e}$, нет влияния изменения массы метеороида на $R_{S}$.

Таким образом, принципиальные отличия разработанных моделей от используемых в литературе следующие. В моделях III и IV учитывается уменьшение плотности облака фрагментов и изменение его формы (влияние $\gamma$ и $k$ ), а в моделях I и II - нет. В моделях III и IV скорость увеличения $R_{S}$ зависит от массы метеороида $M$, которая меняется вследствие абляции, поэтому, чтобы найти $R_{S}$ и $M$, надо решать совместную задачу фрагментации, абляции и торможения метеороида; в моделях I и II задача фрагментации отделена, т.к. $R_{S}$ определяется только начальными, а не текущими параметрами.

\section{3. Решение уравнений метеорной физики. Моделирование абляции}

Для моделирования взаимодействия метеороида с атмосферой используются уравнения физической теории метеоров [3] - уравнения торможения и абляции и уравнения прямолинейной траектории и изотермической атмосферы

$$
\begin{gathered}
M \frac{\mathrm{d} V}{\mathrm{~d} t}=-\frac{\pi}{2} R_{s}^{2} C_{D} \rho V^{2}, \quad Q \frac{\mathrm{d} M}{\mathrm{~d} t}=-\frac{\pi}{2} R_{s}^{2} C_{H} \rho V^{3}, \\
\frac{\mathrm{d} h}{\mathrm{~d} t}=-V \sin \theta, \quad \rho=\rho_{0} \exp \left(-\frac{h}{h^{*}}\right),
\end{gathered}
$$

где $Q$ - эффективная теплота уноса массы; $h$ - высота; $C_{D}$ - коэффициент сопротивления; $C_{H}$ - коэффициент теплопередачи на единицу площади миделева сечения; $\rho_{0}=1.225 \times 10^{-3}$ $\Gamma / \mathrm{cm}^{3}$.

Уравнения (5) решались явным методом Рунге-Кутта 3-го порядка аппроксимации для моделирования взаимодействия Челябинского метеороида с атмосферой с использованием моделей I - IV. До начала разрушения решались уравнения (5) для шара, после уравнения (5) для сфероида решались совместно с тремя уравнениями (1) и (2) для моделей III и IV.

В качестве начальных данных брались наблюдательные данные [9]: $V_{e}=19$ км/с, $\theta=18^{\circ}, \delta_{e}=3.3$ г $/ \mathrm{cm}^{3}$. Неизвестная начальная масса метеороида $M_{e}$ определялась путем воспроизведения наблюдательной кривой энерговыделения [10].

Коэффициент сопротивления сфероида полагался равным $C_{D}=2-1 / k[6,11]$.

Коэффициент радиационной теплопередачи $C_{H}$ для сфероида полагался равным

$$
C_{H}=\psi \varphi \beta C_{H 0}, \quad \varphi=1-\frac{1}{k^{1 / 2}} \frac{8.42-0.17 V}{V-1.454}, \quad \beta=\frac{1}{0.956+0.036 k},
$$

где коэффициент теплопередачи в точке торможения $C_{H 0}$ определялся по формуле работы [12] в зависимости от значений $V, R, \rho ; \beta$ характеризует зависимость теплового потока в точке торможения сфероида от параметра $k[13] ; \varphi-$ изменение его вдоль поверхности [11]. В выражении (6) введен фактор неопределенности $\psi$ для учета влияния на радиационный поток к телу опережающего излучения, экранирования его парами и др. При расчетах $\psi$ варьировался для исследования влияния неопределенности задания $C_{H}$ (включая постоянное его значение, принятое в литературе) на решение. 


\section{4. Результаты расчетов для Челябинского метеороида в рамках разных моделей}

На рис. 1 показано изменение вдоль траектории радиуса миделева сечения и массы раздробленного метеороида для рассмотренных моделей фрагментации. Расчеты проведены при высоте начала разрушения $h_{f}=45 \mathrm{\kappa м}$, что соответствует наблюдательным данным для Челябинского метеороида [9]; $M_{e}$ и $R_{e}$ - значения радиуса и массы метеороида при входе в атмосферу. Неизвестная начальная масса метеороида $M_{e}$ для каждой модели определялась путем корреляции рассчитанного и наблюденного [10] максимума энерговыделения (изменения кинетической энергии на единицу высоты). Так, $M_{e}=(1.1,0.88,1.22,1.6) \times 10^{10}$ г для моделей I, II, III, IV при $C_{H}=0.1$ и $(1.14,0.93,1.32,1.75) \times 10^{10}$ г при $\psi=1$ в формуле (6). Модель III дает значение $M_{e}$, близкое к наиболее вероятным значениям 1.2 и $1.3 \times 10^{10}$ г по оценкам работ [9] и [14]. Модели I и II дают намного большее расширение метеороида в поперечном направлении, чем модели III и IV.
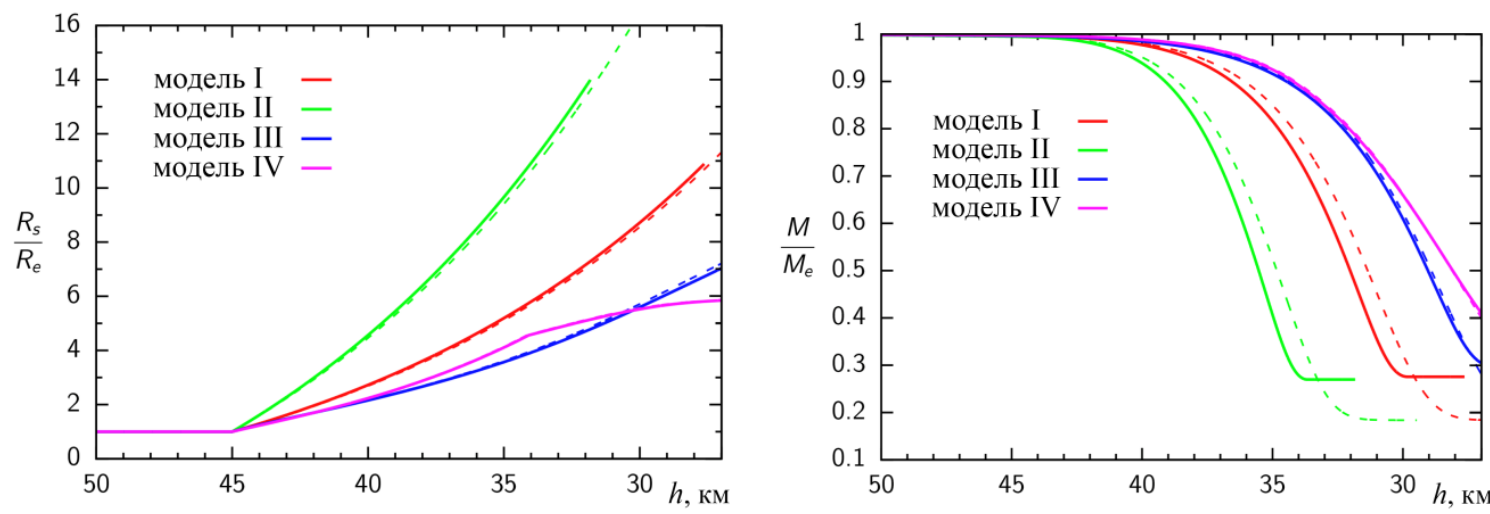

Рис. 1. Изменение радиуса миделева сечения и массы метеороида в зависимости от высоты полета $h$. Сплошные и штриховые кривые - $\psi=1$ в формуле (6) и $C_{H}=0.1$

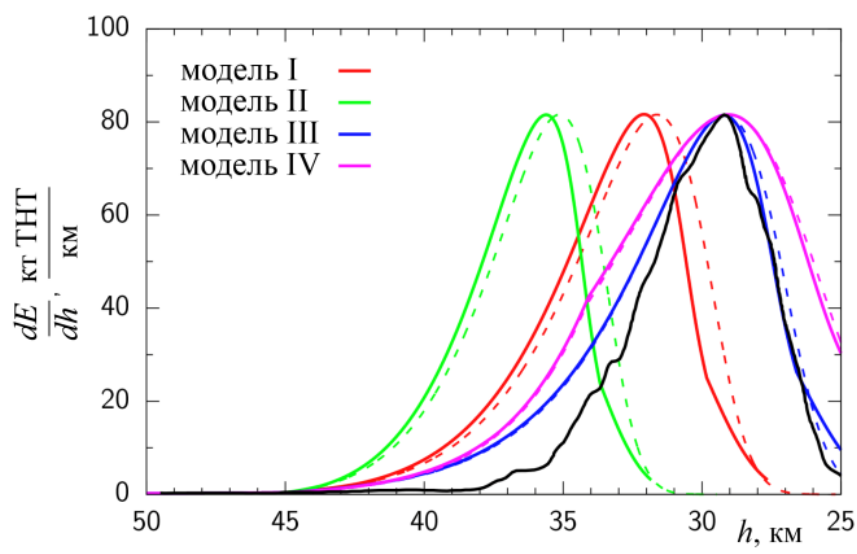

Рис. 2. Энерговыделение. Сравнение с наблюдательными данными [10] - черная кривая

Энерговыделение метеороида на единицу высоты представлено на рис. 2. Здесь же проводится сравнение с наблюдательной кривой энерговыделения [10]. Модели I и II дают высоту пика яркости Челябинского болида на несколько километров выше наблюдаемой, при этом решение с применением модели I ближе к данным наблюдений. Модель III позволяет удовлетворительно смоделировать кривую энерговыделения до высоты 27 км. Решение с применением модели IV хуже согласуется с наблюдательными данными как для кривой энерговыделения, так и для оценки начальной массы, по сравнению с моделью III. 


\section{3. Независимое движение фрагментов. Распределение их по массам}

\section{1. Формулы для распределения фрагментов разрушенного метеороида по массам}

Когда фрагменты метеороида разойдутся на достаточное расстояние, чтобы двигаться независимо, для изучения их движения надо знать их распределение по массам. При этом проводят аналогию с экспериментами по разрушению тел при высокоскоростном ударе.

Результаты многочисленных экспериментов [15-17 и др.] показали, что распределение собранных после катастрофического разрушения фрагментов по массам $m$ хорошо описывается в виде степенного закона

$$
N_{m}=B m^{-\beta}, \text { или } \frac{\mathrm{d} N_{m}}{\mathrm{~d} m}=D m^{-\beta-1}
$$

Здесь $N_{m}$ - кумулятивное число фрагментов с массой больше или равной, чем $m$. Показатель $\beta$ в некоторой степени зависит от материала модели и степени ее дробления (энергии разрушения) [17], типичные значения $\beta$ для скальных пород $0.5 \div 0.7$ [16].

Введем следующие характеристики: $M$ - общая масса всех фрагментов; $m_{l}$ - масса наибольшего фрагмента; $l=m_{l} / M$ - безразмерная масса наибольшего фрагмента; $n_{m}-$ функция распределения, описывающая вероятность нахождения фрагмента массой $m$. Запишем условие сохранения массы всех фрагментов $M$

$$
M=\int_{0}^{m_{l}} n_{m} m \mathrm{~d} m=\int_{0}^{m_{l}} D m^{-\beta} \mathrm{d} m
$$

Используя соотношение (8) и условие $N_{m}\left(m_{l}\right)=1$, найдем решение уравнения (7) в дифференциальной форме (обычно в литературе используется недифференциальная форма), найдем $D$ и получим выражения для $n_{m}$ и $N_{m}$ как функции $m, m_{l}, M$ и показателя степени $\beta$

$$
N_{m}=\frac{1-\beta}{\beta l^{1-\beta}}\left(\left(\frac{m}{M}\right)^{-\beta}-l^{-\beta}\right)+1, \quad n_{m}=\frac{1-\beta}{M l^{1-\beta}}\left(\frac{m}{M}\right)^{-\beta-1}
$$

Полученное кумулятивное распределение фрагментов $N_{m}$ сравнивалось с результатами экспериментов [18] по высокоскоростным соударениям гипсовых шаров, их пористая структура позволяет моделировать разрушение осколков астероидов. На рис. 3 показаны экспериментальные распределения фрагментов по массам для конического (крупные и мелкие частицы, энергия разрушения 3.21 кДж/кг) и катастрофического (мелкие частицы, энергия 45.9 кДж/кг) дроблений, и расчеты $N_{m}$ по формуле (9). Модельные кривые хорошо описывают распределение фрагментов по массам для обоих типов дробления. 


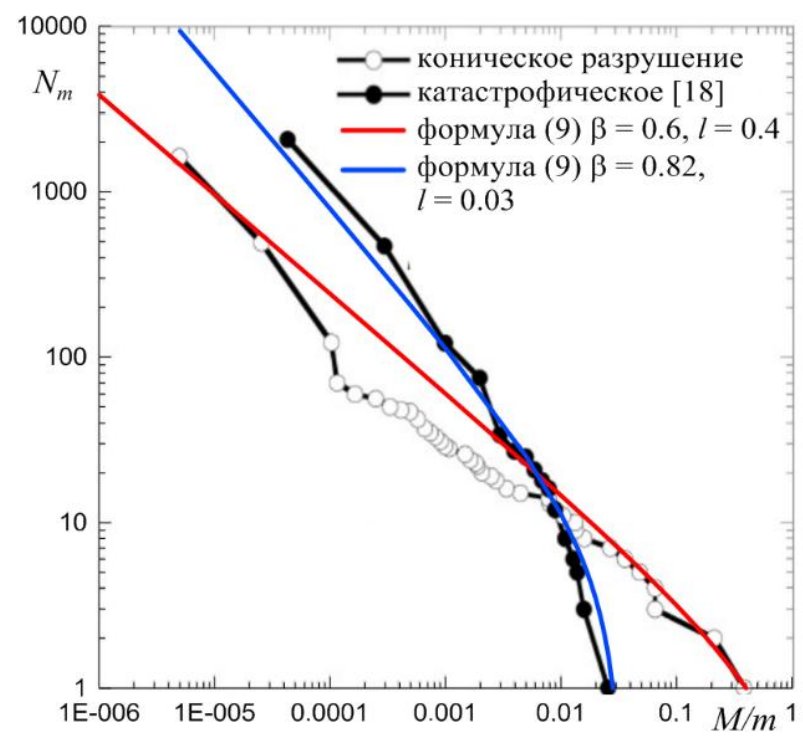

Рис. 3. Сравнение экспериментального кумулятивного распределения фрагментов по массам при коническом и катастрофическом разрушениях гипсовых шаров [18] с расчетами по формуле (9)

\section{2. Сравнение с распределением по массам найденных метеоритов}

Полученное кумулятивное распределение фрагментов по массам сравнивалось также с распределением найденных метеоритов в тех случаях, когда было собрано достаточно большое их количество. На рис. 4, $a$ построено кумулятивное распределение по массам найденных метеоритов после пролета в атмосфере болида Бассикуну 16 октября 2006 г. в Мавритании. Массы 108 фрагментов взяты из каталога [19], масса наибольшего $m_{l}=6.12$ кг, общая масса метеоритов $M=62.375$ кг, $l=0.098$. На рис. 4,6 построено распределение по массам найденных метеоритов после пролета астероида 2008 ТС 37 октября 2008 г. в Судане (Алмахата-Ситта). Массы 662 фрагментов взяты из публикации [20], $m_{l}=0.379$ кг, $M=10.5$ кг, $l=0.036$. На рис. 4, , п построено распределение по массам найденных метеоритов в Словакии после пролета болида Кошице 28 февраля 2010 г. Массы 218 фрагментов взяты из работ [21,22], $m_{l}=2.37$ кг, $M=12.8$ кг, $l=0.185$ (существует большая вероятность, что есть ненайденные фрагменты в лакуне 2167.4-318 г между вторым и третьим [22], поэтому добавлено 3 осколка общим весом 1.5 кг). На рис. 4, 2 распределение по массам найденных метеоритов построено для Челябинского события 15 февраля 2013 г. Массы 1706 фрагментов взяты из работы [14] и базы данных [23], $m_{l}=3.4$ кг, $M=73889$ кг, $l=0.046$.

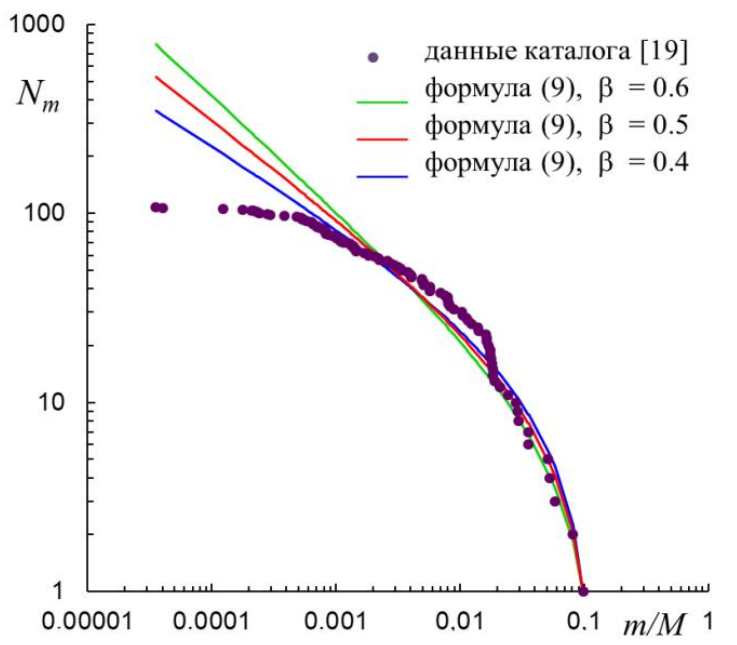

a) Бассикуну

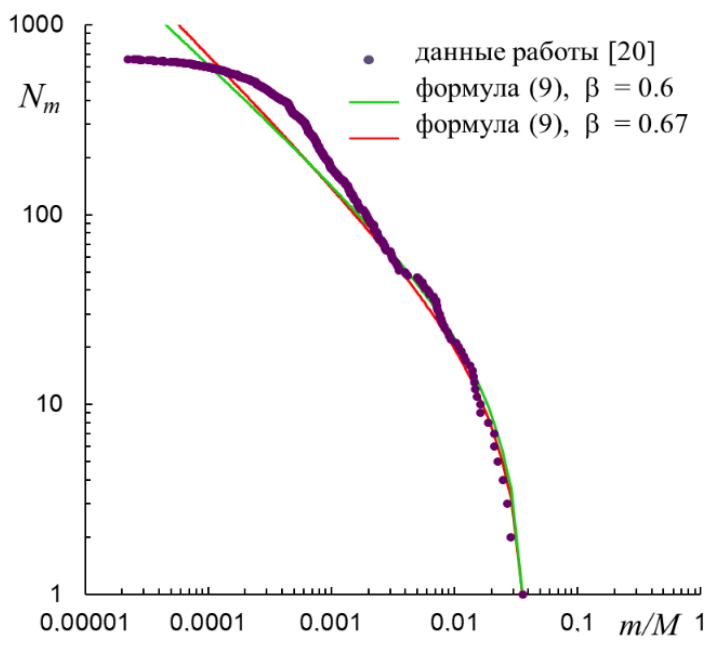

б) Алмахата-Ситта 


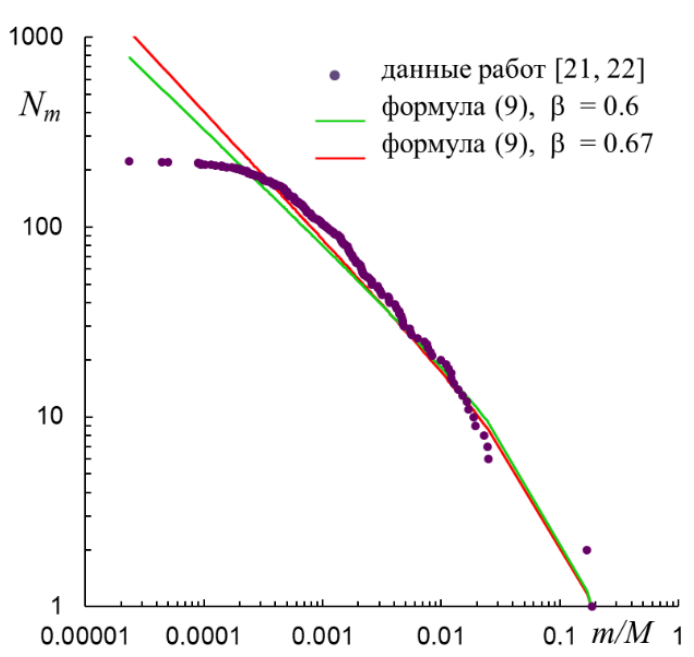

в) Кошице

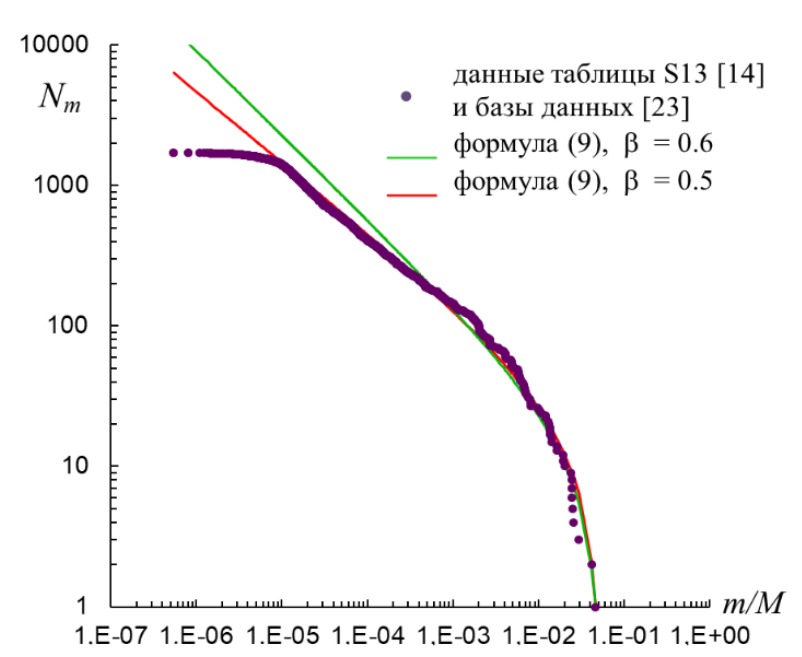

2) Челябинск

Рис. 4. Кумулятивное распределение метеоритов по массам

Из рис. 4 видно, что формула (9) при $\beta=0.6$ удовлетворительно описывают распределения метеоритов для фрагментов с массой более нескольких грамм.

\section{3. Определение уноса массы и светимости роя независимых фрагментов}

Пусть на начальной высоте $h_{0}$ имеется рой независимых фрагментов с вероятностным распределением $n_{m}(9)$ по массам $M_{0}$

$$
n_{m}=\frac{1-\beta}{M_{e} l^{1-\beta}} m_{0}^{-\beta-1}, \quad m_{0}=\frac{M_{0}}{M_{e}}
$$

Здесь $M_{0}$ и $m_{0}$ - нормальная и безразмерная масса фрагмента; $M_{e}$ - общая масса фрагментов на высоте $h_{0} ; l-$ безразмерная масса наибольшего фрагмента.

Для нахождения общей массы разрушенного метеороида $M_{\Sigma}$ и интенсивности его свечения $I_{\Sigma}$ нужно проинтегрировать соответствующие величины по всем фрагментам

$$
\begin{gathered}
M_{\Sigma}=\frac{1-\beta}{l^{1-\beta}} \int_{0}^{l} m_{0}^{-\beta-1} M \mathrm{~d} m_{0}, \\
I_{\Sigma}=-\tau\left(\frac{\mathrm{d} E}{\mathrm{~d} t}\right)_{\Sigma}=\frac{1-\beta}{l^{1-\beta}} \int_{0}^{l} m_{0}^{-\beta-1} \tau\left(-\frac{\mathrm{d}}{\mathrm{d} t} \frac{M V^{2}}{2}\right) \mathrm{d} m_{0}
\end{gathered}
$$

Здесь $\tau$ - коэффициент эффективности излучения. Масса $M$ и скорость $V$ каждого фрагмента в выражениях (11) находятся из уравнений метеорной физики (5) при начальных параметрах: $m_{0}, M_{e}, V_{e}, h_{0}, l$, плотности метеороида $\delta$ и угле входа $\theta$. При постоянных параметре абляции $\sigma=C_{H} /\left(Q C_{D}\right)$ и $C_{D}$ решение уравнений (5) имеет вид

$$
\begin{gathered}
m=m_{0} e^{-\alpha\left(1-v^{2}\right)}, \quad v=\frac{V}{V_{e}}, \quad m=\frac{M}{M_{e}}, \quad \alpha=\frac{\sigma V_{e}^{2}}{2}, \\
E i\left(\alpha^{\prime}\right)-E i\left(\alpha^{\prime} v^{2}\right)=e^{\alpha^{\prime}} m_{0}^{-1 / 3}\left(\rho^{\prime}-\rho_{0}^{\prime}\right), \quad \operatorname{Ei}(x)=v p \int_{-\infty}^{x} \frac{e^{t} \mathrm{~d} t}{t}=0.5772+\ln x+\sum_{k=1}^{\infty} \frac{x^{k}}{k ! k},
\end{gathered}
$$




$$
\alpha^{\prime}=\frac{\alpha}{3}, \quad \rho^{\prime}=\frac{\rho}{\rho_{0} \gamma}, \quad \gamma=\frac{\delta^{2 / 3} M_{e}^{1 / 3} \sin \theta}{f h^{*} C_{D} \rho_{0}}
$$

Здесь $\alpha$ и $\gamma$ - безразмерные параметры; $f$ - формфактор, для сферы $f=1.21$.

\section{4. Заключение}

Разработанные модели, в отличие от используемых в литературе, учитывают изменение плотности и формы разрушенного метеороида. При использовании моделей III, IV надо решать совместную задачу фрагментации, абляции и движения метеороида; в моделях I, II задача фрагментации отделена. Модели III и IV дают значительно меньшее увеличение радиуса миделева сечения метеороида, чем модели I и II.

Модели I и II дают высоту пика яркости Челябинского болида на несколько километров выше наблюдаемой, при этом решение с применением модели I ближе к данным наблюдений. Решение для энерговыделения, полученное с применением модели III, удовлетворительно согласуется с наблюдательными данными до высоты 27 км. На более низких высотах необходимо учитывать движение независимых фрагментов.

Получены формулы для вероятностного и кумулятивного распределений фрагментов разрушенного метеороида по массам. Формула для кумулятивного числа фрагментов удовлетворительно описывает данные экспериментов по высокоскоростным соударениям гипсовых шаров и распределение по массам собранных метеоритов при их выпадении в Кошице, Бассикуну, Алмахата-Ситта (астероид $2008 \mathrm{TC}_{3}$ ) и Челябинске.

Проведенные сравнения дают основание для использования полученных формул при моделировании независимого движения и абляции фрагментов метеороида. Выведены интегральные выражения для уноса массы и светимости разрушенного метеороида с использованием полученного распределения фрагментов по массам.

\section{Благодарности и ссылки на гранты}

Работа выполнена в соответствии с планом исследований НИИ механики МГУ при частичной поддержке гранта РФФИ № 18-01-00740.

\section{Литература}

1. Григорян С.С. О движении и разрушении метеоритов в атмосферах планет// Космич. исслед. 1979. Т. 17. № 6. С. $875-893$.

2. Левин Б.Ю. Физическая теория метеоров и метеорное вещество в солнечной системе. М.: Издво АН СССР, 1956. 293 c.

3. Бронштэн В.А. Физика метеорных явлений. М.: Наука, 1981. 416 с.

4. Григорян С.С., Ибодов Ф.С., Ибадов С.И. Челябинский суперболид: к физике взрыва // Астрон. вестн. 2013. Т. 47. № 4. С. 292-298.

5. Hills J.G., Goda M.P. The fragmentation of small asteroids in the atmosphere // Astron. J. 1993. V. 105. № 3. Pp. 1114-1144.

6. Брыкина И.Г. О модели фрагментации крупного метеороида: моделирование взаимодействия Челябинского метеороида с атмосферой // Астрон. вестн. 2018. Т. 52. № 5. С. 437-446.

7. Artemieva N., Shuvalov V. Motion of a fragmented meteoroid through the planetary atmosphere // J. Geophys. Res. Planets. 2001. V. 106. E2. Pp. 3297-3309.

8. Максимов Ф.А. Сверхзвуковое обтекание системы тел // Компьютерные исследования и моделирование. 2013. № 5. С. 969-980.

9. Borovička J., Spurný P., Brown P., Wiegert P., Kalenda P., Clark, D., Shrbený L. The trajectory, structure and origin of the Chelyabinsk asteroidal impactor // Nature. 2013. V. 503. Pp. 235-237.

10. Brown P.G., Assink J.D., Astiz L., et al. A 500-kiloton airburst over Chelyabinsk and an enhanced hazard from small impactors. // Nature. 2013. V. 503. Pp. 238-241. 
11. Брыкина И.Г., Тирский Г.А. Унос массы и световая кривая крупного метеороида. Аналитическое решение // ПММ. 2017. Т. 81. № 5. С. 571-592.

12. Брыкина И.Г., Егорова Л.А. Аппроксимационные формулы для радиационного теплового потока при больших скоростях // Изв. РАН. МЖГ. 2019. № 4. С. 123-134.

13. Suttles J.T., Sullivan E.M., Margolis S.B. Curve fits of predicted inviscid stagnation-point radiative heating rates, cooling factors, and shock standoff distances for hyperbolic earth entry. NASA TN D7622. 1974. $45 \mathrm{p}$.

14. Popova O.P., Jenniskens P., Emel'yanenko V., Kartashova A., et al. Chelyabinsk Airburst, Damage Assessment, Meteorite Recovery, and Characterization // Science. 2013. V. 342. Iss. 6162. Pp. 10691073. Supplementary Materials (online).

15. Hawkins S. Asteroidal Fragments // Astronomical J. 1960. V. 65. No 5. Pp. 318-322.

16. Hartmann W. K. Terrestrial, lunar, and interplanetary rock fragmentation // Icarus. 1969. V. 10. Pp. 201-211.

17. Fujiwara A., Cerroni, P., Davis, D. R., Ryan, E., Di Martino, M., Holsapple, K., Housen, K. Experiments and scaling laws for catastrophic collisions. // Asteroids II. 1989. Pp. 240-265.

18. Okamoto C., Arakawa M. Experimental study on the collisional disruption of porous gypsum spheres// Meteorit. \& Planet. Sci. 2009. V. 44. Pp. 1947-1954.

19. Buhl S., Baermann M. The Bassikounou Meteorite Fall - Descriptive Catalog of the Recovered Masses. 2007. https://www.meteorite-recon.com/wp-content/uploads/pdf/Buhl Baermann Catalog

20. Shaddad M.H., Jenniskens P., Brown P. et al. The recovery of asteroid 2008 TC3 // Meteorit. \& Planet. Sci. 2010. V. 45. № 10-11. Pp. 1557-1587.

21. Toth J., Svoreň J., Borovička J., Spurný P., et al. The Košice meteorite fall: Recovery and strewn field // Meteorit. \& Planet. Sci. 2015. V. 50. Pp. 853-863.

22. Gritsevich M., Vinnikov V., Kohout T., Toth J., et al. A comprehensive study of distribution laws for the fragments of Košice meteorite // Meteorit. \& Planet. Sci. 2014. V. 49. Pp. 328-345.

23. База данных фрагментов метеорита «Челябинск». https://www.csu.ru/science/chelyabinskmeteor-study-center/database.aspx 